\title{
An effective system for detecting protein-protein interaction based on in vivo cleavage by PPV Nla protease
}

\author{
Nuoyan zheng ${ }^{1,2,3^{*}}$, Xiahe Huang $^{3^{*}}$, Bojiao Yin ${ }^{3}$, Dan Wang ${ }^{4}$, Qi Xie ${ }^{3 凶}$ \\ ${ }^{1}$ Department of Nephrology, the First Affiliated Hospital, Sun Yat-Sen University, Guangzhou 510000, China \\ ${ }^{2}$ Research Center of Translational Medicine, the First Affiliated Hospital, Sun Yat-Sen University, Guangzhou 510000, China \\ ${ }^{3}$ State Key Laboratory of Plant Genomics, National Centre for Plant Gene Research, Institute of Genetics and Developmental \\ Biology, Chinese Academy of Sciences, Beijing 100101, China \\ ${ }^{4}$ Animal Center, Institute of Genetics and Developmental Biology, Chinese Academy of Sciences, Beijing 100101, China \\ $\triangle$ Correspondence: qxie@genetics.ac.cn \\ Received December 19, 2011 Accepted September 25, 2012
}

\begin{abstract}
Detection of protein-protein interaction can provide valuable information for investigating the biological function of proteins. The current methods that applied in protein-protein interaction, such as co-immunoprecipitation and pull down etc., often cause plenty of working time due to the burdensome cloning and purification procedures. Here we established a system that characterization of protein-protein interaction was accomplished by co-expression and simply purification of target proteins from one expression cassette within $E$. coli system. We modified $p E T$ vector into co-expression vector plnvivo which encoded PPV Nla protease, two cleavage site $F$ and two multiple cloning sites that flanking cleavage sites. The target proteins (for example: protein A and protein B) were inserted at multiple cloning sites and translated into polyprotein in the order of MBP tag-protein A-site F-PPV Nla protease-site F-protein B-His 6 tag. PPV Nla protease carried out intracellular cleavage along expression, then led to the separation of polyprotein components, therefore, the interaction between protein A-protein B can be detected through one-step purification and analysis. Negative control for protein B was brought into this system for monitoring interaction specificity. We successfully employed this system to prove two cases of reported protien-protein interaction: RHA2a/ANAC and FTA/FTB. In conclusion, a convenient and efficient system has been
\end{abstract}

successfully developed for detecting protein-protein interaction.

KEYWORDS PPV Nla protease, protein-protein interaction, in vivo cleavage, fusion protein

\section{INTRODUCTION}

The interaction between proteins has numerous effects on cellular process in the cell: protein folding, enzyme kinetics; substrate channeling; cell-cycle control, cell differentiation, downstream signaling etc. (Phizicky and Fields, 1995). The types of protein-protein interaction can basically be classified into stable and transient interaction. There have been numerous approaches for detecting protein-protein interaction including high throughput methods such as yeast two-hybrid, phage display, tandem affinity purification system, protein microarray etc. and low throughput methods such as pull-down assay, co-immunoprecipitation, confocal microscopy, surface plasmon resonance, label transfer and fluorescence resonance energy transfer etc. (Chautard et al., 2009; Kerrigan et al., 2011). Pull-down assay was broadly applied in detecting stable physical interaction between proteins in vitro due to its high specificity and desirable feasibility. The common requirement for pull-down system is the availability of a purified bait protein linked to affinity support and a prey protein in forms of purified product, in vitro-translation product or cell lysate etc., thus plenty of work is needed for cloning and optimization for expression and purification.

*These authors contributed equally to the work. 
When post-modification is not needed, the $E$. coli expression system was commonly used in pull-down assay due to easy manipulation and high yield as compared with original host. Under most circumstances, the prey and bait protein were separately expressed and reconstituted in vitro, which caused additional workload for expression and purification optimization. Meanwhile, some proteins were impaired regarding of biological activity, correct folding, and interacting capability after elaborate steps of manipulation along expression and purification. Another concern is that some interacting proteins only became soluble when all partners were presented together (Kummel et al., 2005; Tundup et al., 2006). New methods based on co-expression strategy offered alternative way to facilitate the process of protein expression and protein-protein interaction, by using compatible vectors (Huppa and Ploegh, 1997; Fabian et al., 1998; Hanzlowsky et al., 2006), multiple promoters (Belyaev and Roy, 1993; Scheich et al., 2007), polycistrons (Selleck et al., 2005; Neumann et al., 2007; Bieniossek et al., 2009), fusion linkers (Clements et al., 2000) in both prokaryotic and eukaryotic systems. One advantageous application was that TEV (Tobacco Etch Virus) Nla protease carried out site-specific cleavage on polyprotein to yield multiple native proteins within $E$. coli using a single promoter (Shih et al., 2005; Chen et al., 2010). TEV and PPV (Plum Pox Virus) belong to Potyviridae family with similar proteolytic function (Urcuqui-Inchima et al., 2001), therefore we speculated that PPV Nla protease can act as a powerful tool in vivo to process co-expressed substrate and then facilitate the verification of protein-protein interaction.

Here we aimed to establish a system that tagged prey protein, PPV Nla protease, tagged bait protein, can be co-expressed as polyprotein from a single expression cassette within E. coli. After co-expression, these two candidate proteins were cleaved by PPV Nla protease at specific site $\mathrm{F}$ and then become independent components within the cell. Consequently, the possible protein-protein interaction can be detected by one-step purification in appropriate buffer. The essential prerequisite of this system is the highly effective proteolytic action of PPV Nla protease in generating spliced components within the $E$. coli system. To fulfill these goals, we first confirmed that intracellular cleavage of PPV Nla protease on polyprotein can result in independent separated components, and then we used this co-expression system to verify two cases of protein-protein interaction: RHA2a/ANAC and FTA/FTB. Our result showed that PPV Nla protease can greatly facilitate the detection of protein-protein interaction within one expression system, and no faulse positive interaction was observed.

\section{RESULTS}

Though we previously reported the in vitro proteolytic action of PPV Nla protease on recombinant substrates (Zheng et al., 2008), no direct evidence show that PPV Nla protease can carry out intracellular cleavage for self-containing artificial polyprotein within E. coli, as TEV Nla protease did (Shih et al., 2005; Chen et al., 2010). Here we constructed an expression cassette in which EGFP protein was expressed downstream of PPV Nla protease and site $F$, therefore the polypeptide composed of Tag-Protease-site F-EGFP (designated as P-sG) was transcribed within E. coli (Fig. 1A). SDS-PAGE analysis of $E$. coli lysate showed that P-sG polyprotein split into two components with sizes respectively identical to those of Tag-Protease and EGFP in SDS-PAGE, whereas no protein corresponding to $\mathrm{P}$-sG polyprotien in size was detected after induction (Fig. 1B). This indicated the proteolysis event happened quickly or even simultaneously along expression, and more importantly, this process was very efficient since polyprotein P-sG was not distinguishable in SDS-PAGE. Furthermore, the $p E T$-P-sG-tranformed $E$. coli culture emitted strong green fluorescence under UV microscopy, while control sample of $p E T-P$ which only contain protease but lack of EGFP were not fluorescent (Fig. 1C). This demonstrated intracellular proteolysis event did not affect the native properties of EGFP protein derived from $p E T-P$-sG.

The fulfillment of in vivo cleavage by PPV Nla protease offered the possibility to employ a single promoter to express multiple candidate proteins and examine their interaction within the E. coli system in our study. As shown in Fig. 2A, plnvivo vector offers two multiple cloning sites (MCS I and MCS II) respectively for protein A (bait protein) and B (prey protein) cloning, while the control plnvivo-Trx vector offers the MCS I for protein A cloning and Trx protein was used as control for protein B. As driven by the powerful $\mathrm{T} 7$ promoter, a long polypeptide chain containing multiple components was produced. Afterwards, PPV Nla protease can self-process the polyprotein into separated components: MBP-Protein A, PPV Nla protease, Protein B-His 6 . Once MBP-Protein A was purified by amylose resin, protein $\mathrm{B}_{-} \mathrm{His}_{6}$ may co-precipitate along the purification process if protein $A$ and $B$ have interaction and buffer environment allows (Fig. 2B).

We applied Arabidopsis RING-finger protein RHA2a and a plant-specific NAC family member ANAC protein as bait-prey pair to test the feasibility of this system. It had been proved that recombinant RHA2a and ANAC protein purified from $E$. coli were positively associated in pull-down assay and yeast two-hybrid assay. Moreover, they found that truncated RHA2a (38-155 aa) protein lacking the putative RHA2a $\mathrm{N}$-terminal transmembrane domain bound to the conserved NAC domain of ANAC protein (1-168 aa) (Krestine et al., 2003). In our system, RHA2a (38-155 aa) and ANAC (1-168 aa) played as protein $A$ and $B$ and the resulted polyprotein was referred as RHA2a-sPs-ANAC, while Trx tag replaced ANAC domain in control expression cassette and the resulted polyprotein was referred as RHA2a-sPs-Trx. After IPTG induction, the harvested $E$. coli cells were subjected to sonication in native buffer, and then the supernatant was subjected to MBP purification followed by adequate washing. Fractions 
A

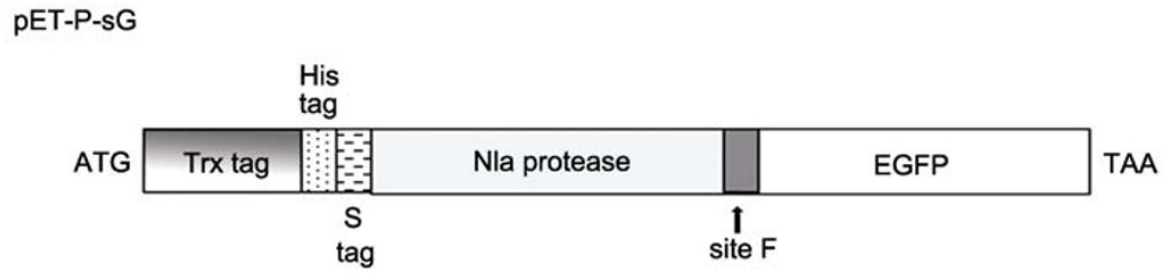

B

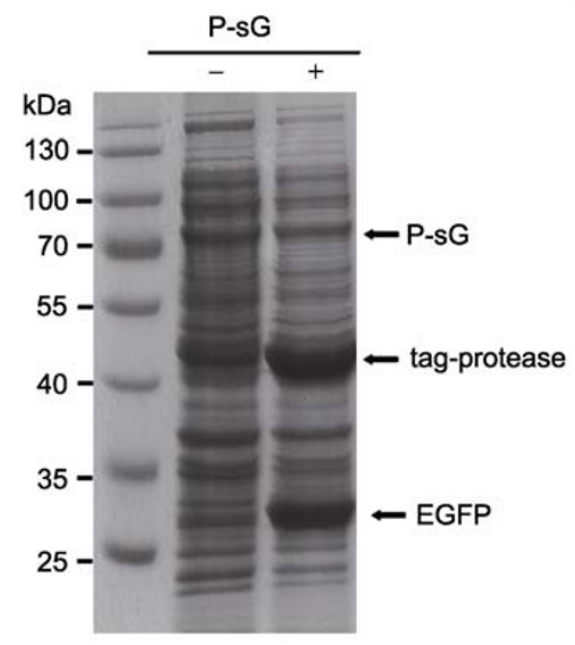

C

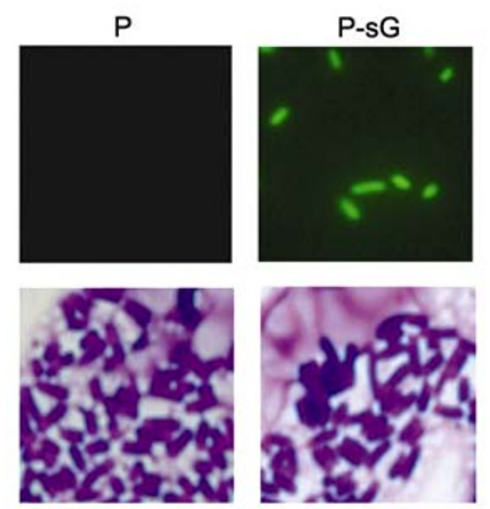

Figure 1. The in vivo cleavage of P-sG polyprotein by PPV Nla protease. (A) The schematic diagram of P-sG polyprotein expression cassette. (B) The SDS-PAGE analysis of P-sG expression and proteolysis. "-": before induction; "+": after induction. The IPTG-induced culture was resuspended in PBS and then directly subjected to SDS-PAGE analysis. Each lane was from $0.1 \mathrm{~mL}$ of harvested $E$. coli culture. (C) The $E$. coli culture after induction was subjected to UV fluorescence observation (upper panel) or gram stain followed by oil-immersion observation (lower panel). P represented PPV Nla protease expressed from $p E T-P$; P-sG represented Tag-Protease-site F-EGFP fused protein expressed from $p E T-P$-sG.

from each step along the process were collected and analyzed by SDS-PAGE and Western blot. The result (Fig. 3A-a, lane 2/5) showed that after induction and expression, the long polyprotein was divided into three components: MBP-RHA2a (57 kDa); PPV Nla protease (29 kDa); ANAC-6×His (24 kDa) or Trx-6xHis (14 kDa). In the lysate supernatant, the solubility of MBP-RHA2a component was less than Protease, ANAC$6 \times$ His, Trx-6×His components (Fig. 3A-a, lane 3/6). However, the MBP-RHA2a fragment was successfully enriched and purified by MBP binding resin (Fig. 3A-a-pull down). In line with our expectation, a band with size of ANAC- $6 \times$ His fragment was detected in the amylose-binding complex as shown in SDS-PAGE (Fig. 3A-a-pull down(1)). A faint band corresponding to the size of protease was also detected in pull-down deposit, which may be caused by the dynamic formation of protease-substrate complex during or even after proteolytic action. The identity of MBP-RHA2a and ANAC-6xHis were separately confirmed by anti-MBP antibody and anti-6xHis antibody in Western blot (Fig. 3A-b). In the control polyprotein RHA2a-sPs-Trx, the pull-down complex contained no detectable Trx-6×His component in either SDS- PAGE (Fig. 3A-a-pull down (2)) or sensitive Western blot assay (Fig. 3A-b), even though the yield of Trx-6xHis His protein in supernatant was higher than that of ANAC- $6 \times \mathrm{His}$ protein (Fig. 3A-a, lane 3/6). This result indicated the interaction between RHA2a and ANAC was specific and did not result from unspecific binding.

Another case of interaction is between Arabidopsis farnesyltransfereas $\alpha$ subunit (FTA) and $\beta$ subunit (FTB), which had shown interaction in yeast two-hybrid (Caldelari et al., 2001) and bimolecular fluorescence complementation assay after transient expression in tobacco cells (Bracha-Drori et al., 2004). In Fig. 3B, the MBP-FTA (83 kDa), Protease (29 kDa),

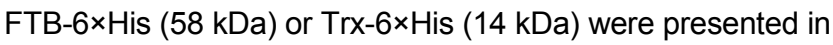
the soluble fraction as indicated by SDS-PAGE and Western blot. MBP-FTA component was easily purified with amylose resin without unspecific binding (Fig. 3B-a-pull down). Only FTB- $6 \times$ His but not Trx-6xHis fragment co-precipitated with 
A
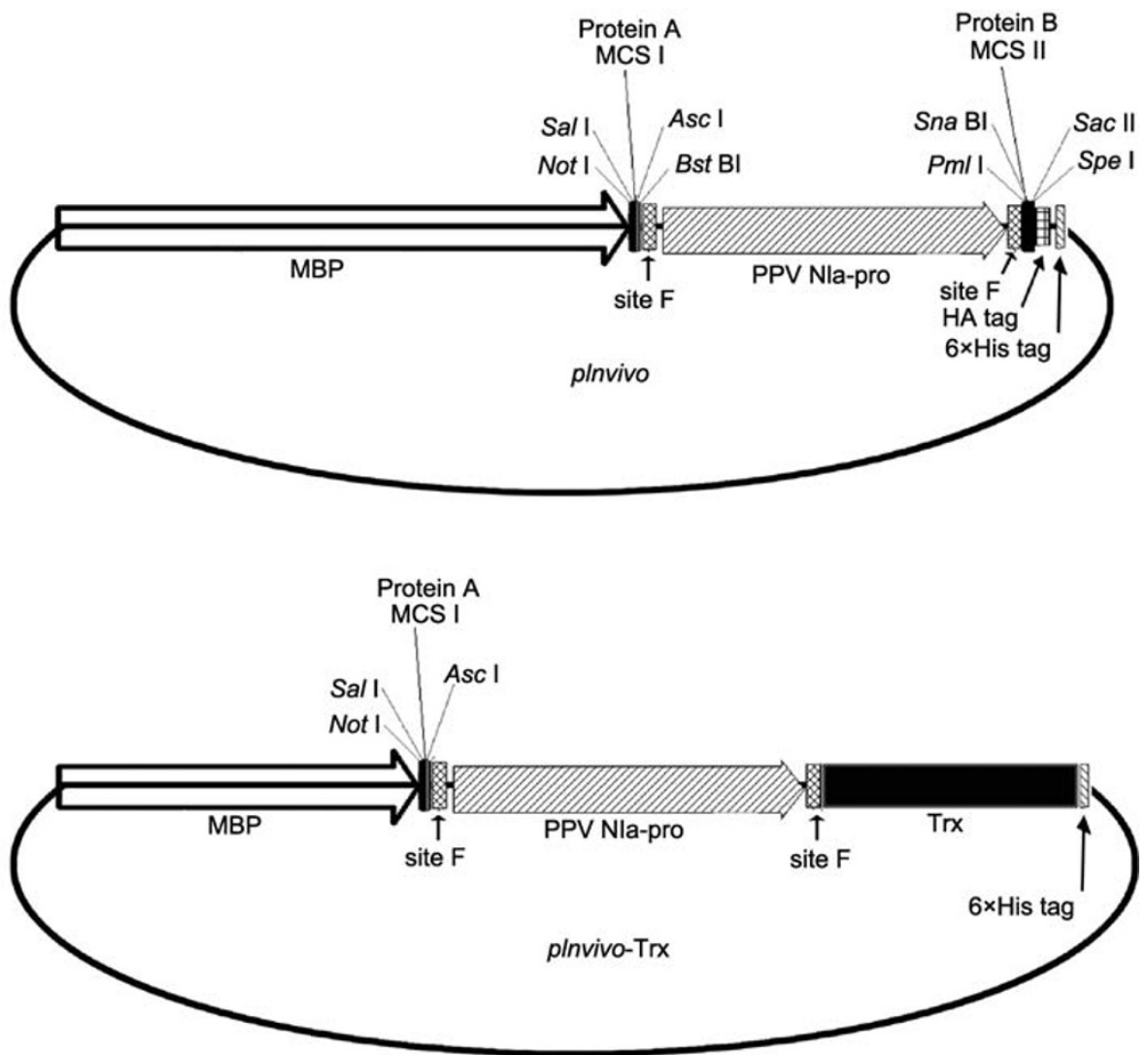

B
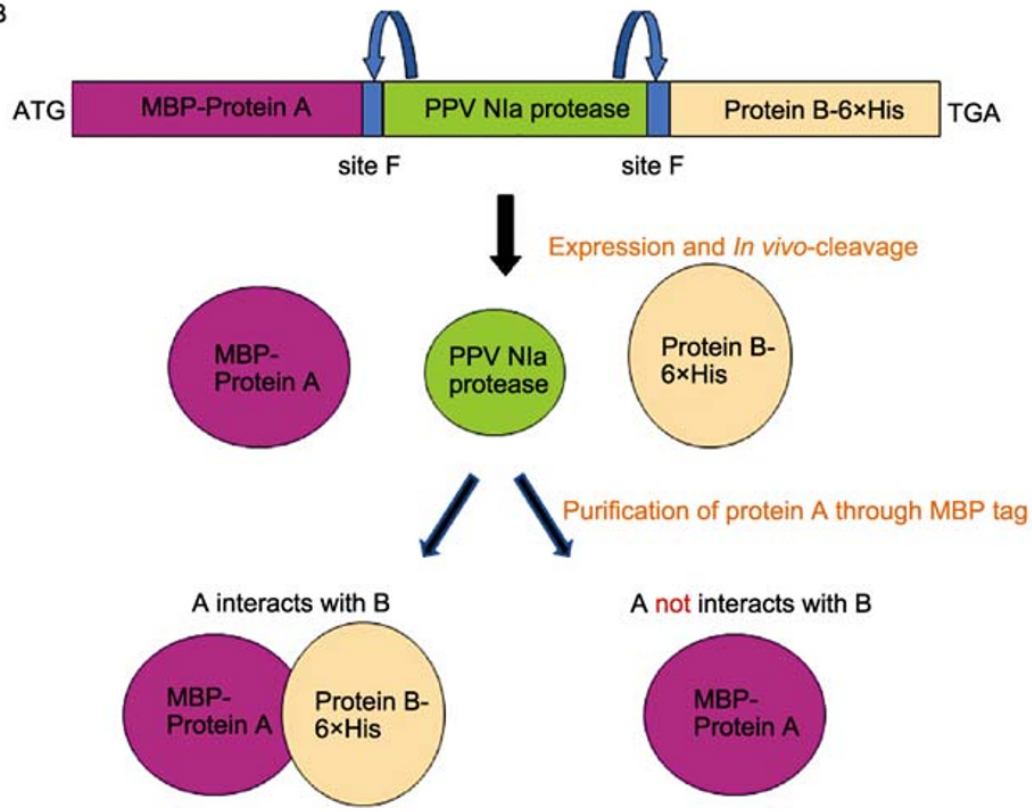

A not interacts with B

Figure 2. The illustration of constructs and procedure for detecting protein-protein interaction in our system. (A) The schematic diagram of coding region in plasmid plnvivo and plnvivo-Trx. The plasmid plnvivo was modified from prokaryotic expression vector $p E T-28 a(+)$ with $\mathrm{T7}$ promoter for the polyprotein expression. The expression cassette contains nucleotide encoding for MBP tag, MCS I, site F, PPV Nla protease, site F, MCS II, and 6xHis tag in the direction from 5'- to 3'-end. plnvivo-Trx lacked the MCS II site and Trx tag was used as negative control for protein B. (B) The graphic process designed for detecting protein-protein interaction from expression to purification. 
A
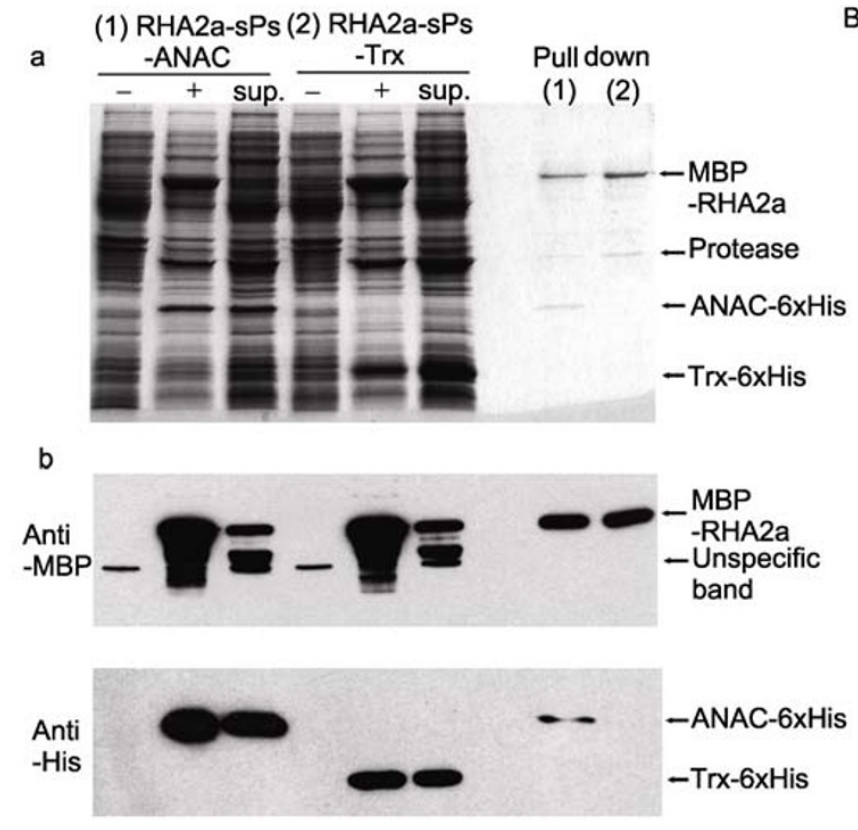

B

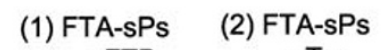

a

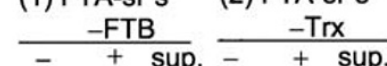

Pull down

(1) (2)

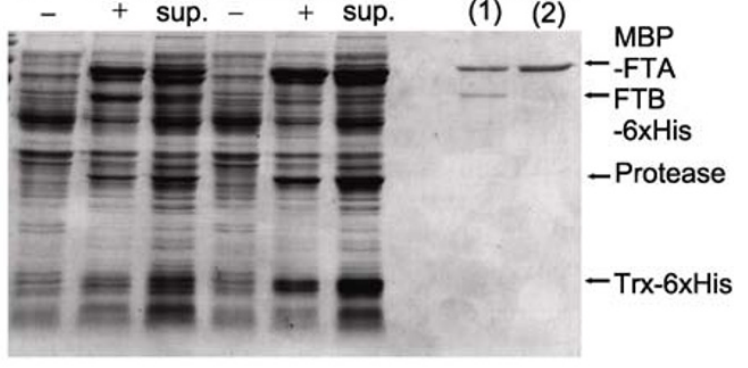

b
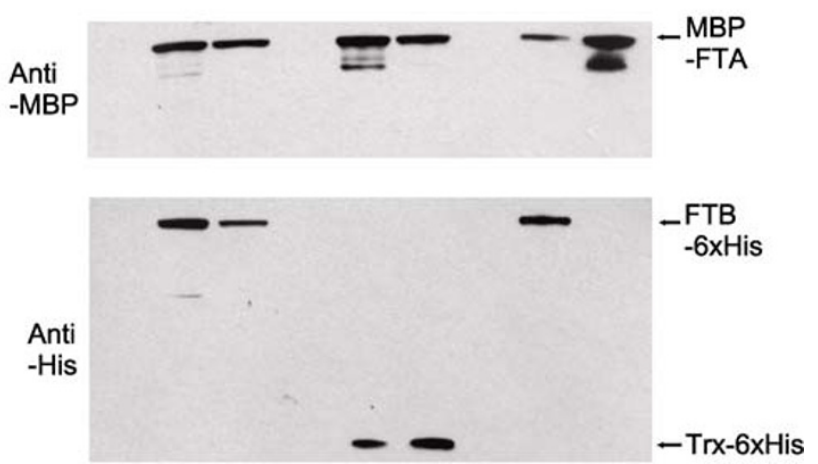

Figure 3. Detecting protein-protein interaction in our system based on in vivo cleavage of PPV Nla protease. (A) (a) The SDS-PAGE analysis of RHA2a and ANAC interaction along expression and purification. RHA2a-sPs-ANAC: polyprotein contained MBP-RHA2a-siteF-protease-siteF-ANAC-His 6 ; RHA2a-sPs-Trx: polyprotein contained MBP-RHA2a-siteF-protease-siteF-Trx-His ${ }_{6}$. "-": before induction; "+": after induction; "sup.": supernatant; pull down (1): Amylose pull-down complex from polyprotein RHA2a-SPs-ANAC; pull down (2): Amylose pull-down complex from polyprotein RHA2a-sPs-Trx; The arrows indicated different cleaved components from polyprotein. (b) The Western blot analysis for RHA2a and ANAC interaction along expression and purification. The specificity of MBP-RHA2a was indicated by anti-MBP antibody while ANAC- $6 \times$ His, and Trx-6×His were respectively was detected by anti-6xHis antibody. (B) Detecting the interaction between FTA and FTB from polyprotein FTA-sPs-FTB and FTA-sPs-Trx with identical process as (A).

MBP-FTA after MBP tag purification, in both SDS-PAGE and Western blot analysis (Fig. 3B-pull down). Though the size of polyprotein FTA-sPs-FTB was up to $170 \mathrm{kDa}$, the whole process along expression, proteolytic cleavage, and purification was surprisingly efficient.

Since we used MBP tag to facilitate the expression and purification of protein $A$, the interaction of MBP-protein $A$ and protein $\mathrm{B}-\mathrm{His}_{6}$ may be due to the false interaction between MBP and protein $\mathrm{B}$. To exclude this possibility, binding capacity of MBP/ANAC-His 6 and MBP/FTB-His 6 were analyzed. The expressed and lysed products from $p E T-M B P-s P s-A N A C$ and $p E T$-RHA2a-sPs-ANAC were subjected to amylose purification and Western blot analysis. As we confirmed that ANAC-His 6 can bind and precipitate together with MBP-RHA2a (Fig. 4, lane 2), ANAC-His 6 did not bind to MBP tag itself (Fig. 4, lane 1). Similar result was achieved in the case of $p E T-M B P$ - $s P s-F T B$ and $p E T-F T A-s P s-F T B$. Positive binding was observed between components MBP-FTA and FTB-His ${ }_{6}$ (Fig. 4, lane 4), but not between MBP and FTB-His 6 (Fig. 4, lane 3).

\section{DISCUSSION}

TEV and PPV are members of the Potyviridae family, and Nla proteases from different potyviruses are highly homologous considering sequence conservation and proteolytic function except that they recognized and cleaved at distinct sevenamino acid sites (Himmler et al., 1990; Urcuqui-Inchima et al., 2001). The proteolytic capacity of PPV Nla protease had long been acknowledged (Garcia et al., 1989), though much less commercialized as TEV protease. Our previous report had proven that recombinant PPV Nla protease can efficiently and specifically cleave fusion protein at recognition site $F$ (NVVVHQ $\downarrow$ A) in vitro (Zheng et al., 2008). A more recent report showed that TEV protease was capable of processing self-containing polyprotein within $E$. coli and mammalian cells to produce separate and stoichiometric components with appropriate intracellular localization, as well as capable of producing binding complex (Chen et al., 2010). Here, we showed that PPV Nla protease was also capable of proc- 

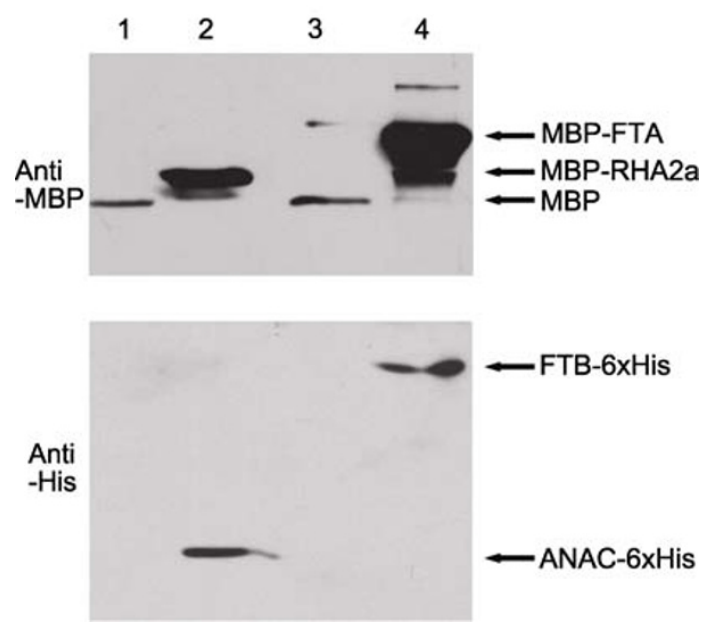

Figure 4. Analysis of binding capacity of MBP fusion tag to candidate protein B. Plasmid $p E T-M B P-s P s-A N A C$ (lane 1), pET-RHA2a-sPs-ANAC (lane 2), pET-MBP-sPs-FTB (lane 3), pET-FTA-sPs-FTB (lane 4) were transformed into BL21(DE3) and induced for protein expression. The sonicated supernatant was subjected to MBP purification and further Western blot analysis.

essing self-containing artificial polyprotein expressed from a single promoter in vivo, yielding multiple separated components. Furthermore, we used this beneficial character of PPV Nla protease to establish a system specially for detecting protein-protein interaction. The intracellular cleavage conducted by PPV Nla protease was complete, since no sign of uncleaved polyproteins was detected in Western blot in all our cases.

The yield and stoichiometry of different components are key determinants to evaluate a system that aimed to express multiple components within the same cell. Multiple vectors strategy led to inefficient expression and difficulty of transfection, while multiple promoters or polycistron strategy may result in suppressed down-stream transcription or undesirable expression proportions (Villemure et al., 2001; Dzivenu et al., 2004; Patial et al., 2007; Chen et al., 2009). We overcame this impediment since multiple components came from a single long transcript and accumulated to high level with appropriate proportions in our system. We speculated that simultaneous translation along transcription in prokaryotic system contributed to quick synthesis and lysis action of PPV Nla protease on substrates, and finally resulted in good tolerance for long transcript and transcribed product in our system.

Detecting protein-protein interaction based on co-expression strategy is appreciated due to simplicity and convenience. However, negative control should be included to avoid false positive binding due to intracellular spatial proximity and possible incomplete processing. We provided ready-to-use vector plnvivo for prey and bait proteins cloning and co-expression, while plnvivo-Trx vector was employed as negative control for prey protein. Besides, we showed that
MBP tag did not bind to RHA2a and FTB. All these data proved that no false interaction was presented in this system.

Through the cases, we can come to a conclusion that this system is quite time-saving and easy-handling, without false positive interaction observed. The benefits of the system includes: (1) Simple cloning strategy and manipulation procedure for target proteins by using plnvivo and plnvivo-Trx vectors; (2) Strong, efficient and simultaneous expression of bait and prey proteins through one promoter, with good tolerance for protein with large size; (3) The fast and feasible detection of protein-protein interaction by one-step purification and analysis, which is beneficial for protein activity and function. The detection for protein-protein interaction can be accomplished in SDS-PAGE analysis with no need of Western blot analysis in this system, which was contributed by high expression amount and stringent negative control. Due to the limit of prokaryotic expression system, plnvivo and plnvivo-Trx plasmids are not suitable for detecting protein-protein interaction with demand of post-modification. However, we can modify these expression cassettes for use in bacluovirus insect cells or mammalian cells by adopting suitable backbones and transcriptional elements.

\section{MATERIALS AND METHODS}

\section{Reagents and materials}

The $p E T-28 a(+), p E T-32 a(+)$ vectors were purchased from EMD chemicals (Madison, Wisconsin, USA); The rabbit anti-MBP tag monoclonal antibody and the mouse anti-His tag antibody were purchased from Sigma (St. Louis, Missouri, USA) and EMD Millipore Chemicals (Darmstadt, Germany), respectively. The amylose resin and $p M A L-C 2 X$ vector were purchased from New England Biolabs company (Ipswich, MA, USA). The reagents used for molecular cloning were bought from TAKARA (Otsu, Japan).

\section{Plasmids construction}

To generate expression vector $p E T-P$-sG, the coding sequence for PPV Nla protease domain was amplified by PCR from $p E T-P$ plasmid and inserted into $p E T$-sG which contain EGFP coding sequence as described in our previous work (Zheng et al., 2008). As a consequence, PPV Nla protease was downstream of EGFP, while cleavage site $\mathrm{F}$ was inserted between PPV Nla protease and EGFP protein.

The construction of expression vectors $p E T$-RHA2a-sPs- ANAC and $p E T-R H A 2 a-s P s-T r x$ was described briefly as follows. The Arabidopsis genes RHA2a (At1g15100) was amplified from total Arabidopsis cDNA to harvest the 114-465 bp cDNA fragment. This amplified fragment and synthesized short linker containing site $\mathrm{F}$ were inserted into $P M A L-C 2 X$ vector right after MBP fusion tag sequence, yielding a new plasmid pMAL-MBP-RHA2a-sF. The DNA fragment encoding PPV Nla protease and site $\mathrm{F}$ from plasmid $p E T-P$-sG plasmid was cloned into $p E T-28 a(+)$ vector to generate the new plasmid $\mathrm{p} E T-28 \mathrm{a}(+)-$-Pro-sF. Fragment encoding MBP-RHA2asite $\mathrm{F}$ was cut from $\mathrm{PMAL-MBP}-\mathrm{RHA2a-SF}$ and cloned into 
$p E T-28 a(+)-P r o-s F$ to generate $p E T-R H A 2 a-s P s$ plasmid. ANAC gene (At1g52890.1) was amplified from total Arabidopsis cDNA to harvest the 1-504 bp cDNA fragments, while the control Trx cDNA fragment was amplified from $p E T-32 a(+)$ vector. Afterwards, ANAC or control thioredoxin (Trx) DNA fragment was cloned into pET-RHA2a-sPs before the $6 \times$ His sequence to generate pET-RHA2a-sPs-ANAC or pET-RHA2a-sPs-Trx plasmid.

The expression vector plnvivo for cloning different bait-prey pairs was generated by replacing RHA2a and ANAC DNA fragment respectively with short linkers MCS I and MCS II in pET-RHA2a$s P s-A N A C$ plasmid. The control vector plnvivo-Trx was made by only replacing RHA2a region with MCS I in pET-RHA2a-sPs-Trx plasmid.

The $\mathrm{p} E T-F T A-s P s-F T B$ was generated by inserting full length FTA (At3g59380) and FTB (At5g40280) cDNA fragment into plnvivo vector respectively at MCS I (Notl-Sall) and MCS II (SnaBI-Spel); while pET-FTA-sPs-Trx contained FTA fragment was achieved by inserting FTA fragment into plnvivo-Trx vector at MCS I (Notl-Sall).

To rule out false positive interaction between fusion tag MBP and protein $\mathrm{B}, p E T-M B P-s P s-A N A C$ and $p E T-M B P$ - $s P s-F T B$ were generated. The ANAC and FTB fragment were amplified with flanking enzymes sites at the $5^{\prime}$ end and $3^{\prime}$ end (SnaBI and Spel), and then inserted into plnvivo vector after endonuclease digestion.

All the clones with inserts in correct orientation were confirmed with restriction enzyme digestion analysis and DNA sequencing.

\section{Expression and extraction of target proteins from E. coli}

The recombinant plasmids were individually transformed into $E$. coli strain BL21 (DE3), and were grown overnight in LB medium supplemented with $100 \mu \mathrm{g} / \mathrm{mL}$ ampicillin at $37^{\circ} \mathrm{C}$. The overnight culture was diluted until absorbance $A_{600 \mathrm{~nm}}=0.1$ and cultured at $28^{\circ} \mathrm{C}$. When the culture reached the mid-log phase $\left(A_{600 \mathrm{~nm}}=0.4\right)$, glucose was added into the medium at a final concentration of $0.2 \%$. An hour later, IPTG was added at a final concentration of $0.25 \mathrm{mmol} / \mathrm{L}$ and prolonged for another $4 \mathrm{~h}$. The cells were harvested and washed with PBS. Each of $50 \mathrm{~mL}$ E. coli culture was resuspended and homogenized in $3 \mathrm{~mL}$ ice-cold lysis buffer, followed by sonication. The homogenates were centrifuged at $12,000 \mathrm{rpm}$ at $4^{\circ} \mathrm{C}$ for $30 \mathrm{~min}$ to remove the cell debris. The lysis buffer was composed of $50 \mathrm{mmol} / \mathrm{L}$ Tris-Cl, $250 \mathrm{mmol} / \mathrm{L} \mathrm{NaCl}, 2 \mathrm{mmol} / \mathrm{L} \mathrm{MgCl}_{2}, 0.2 \%$ Tween-20, $10 \%$ glycerol, $2 \mathrm{mmol} / \mathrm{L}$ DTT with with fresh-made protease inhibitor containing $1 \mathrm{mmol} / \mathrm{L}$ PMSF and $1 \mathrm{mg} / \mathrm{mL}$ benzamidine.

\section{In-vitro purification and detection of target proteins}

For P-sG polyprotein expression and detection, the lysed sample was subjected to SDS-PAGE analysis. Meanwhile, the original E. coli culture was also observed under UV fluorescence microscopy and immersion oil microscopy (DM5000B, LEICA).

To verify RHA2a/ANAC and FTA/FTB interaction, $200 \mu \mathrm{L}$ of the lysed supernatant was incubated with $50 \mu \mathrm{L}$ of amylose resin in 1.0 $\mathrm{mL}$ of binding buffer for $2-4 \mathrm{~h}$ at room temperature to capture the MBP-tagged protein. The binding buffer is very similar to the lysis buffer except that the final concentration of $\mathrm{NaCl}$ was adjusted to a suitable concentration in the range from 100-300 mmol/L. according to the interacting strength between the prey and bait protein. The precipitated complex was washed with $1 \mathrm{~mL}$ of binding buffer for 4-6 times to remove unbound proteins. The residual sediment was resuspended in $45 \mu \mathrm{L}$ of $1 \times$ SDS-PAGE sample buffer, heated at $95^{\circ} \mathrm{C}$ for $15 \mathrm{~min}$ to disassemble the pull-down complex. The subsequent supernatant was subjected to SDS-PAGE and Western blot analysis. For RHA2a/ANAC interaction, $0.1 \mathrm{mmol} / \mathrm{L} \mathrm{ZnCl}_{2}$ was included in $E$. coli culture and binding buffer in order to maintain the structural stability of RHA2a ring finger protein.

To investigate the possible interaction between MBP tag and candidate protein $B$, we generated control plasmids to investigate the interaction of MBP/ANAC-His ${ }_{6}$ and MBP/FTB- His $_{6}$. The expression and purification procedure was conducted as described above.

\section{ACKNOWLEDGMENTS}

We thank Professor Juan Antonio Garcia for providing plasmid pGGNla which contain the cDNA region of PPV Nla protease. This research was supported by National Natural Science Foundation of China (Grant No. 31030047) and the National Basic Research Program (973 Program) (No. 2011CB915402).

\section{ABBREVIATIONS}

ANAC, Arabidopsis NAC domain containing protein 19; co-IP, co-immunoprecipitation; DTT, dithiothreitol; FTA, farnesyltransferase subunit $\alpha$; FTB, farnesyltransferase subunit $\beta$; IPTG, isopropyl-b-D-thiogalactopyranoside; MBP, maltose binding protein; MCS, multiple cloning sites; PMSF, phenylmethanesul -fonylfluoride; PPV, plum pox virus; RHA2a, RING-H2 finger protein RHA2a; TEV, tobacco etch virus; Trx, thioredoxin

\section{REFERENCES}

Belyaev, A.S., and Roy, P. (1993). Development of baculovirus triple and quadruple expression vectors: co-expression of three or four bluetongue virus proteins and the synthesis of bluetongue virus-like particles in insect cells. Nucleic Acids Res 21, 1219-1223.

Bieniossek, C., Nie, Y., Frey, D., Olieric, N., Schaffitzel, C., Collinson, I., Romier, C., Berger, P., Richmond, T.J., Steinmetz, M.O., et al. (2009). Automated unrestricted multigene recombineering for multiprotein complex production. Nat Methods 6, 447-450.

Bracha-Drori, K., Shichrur, K., Katz, A., Oliva, M., Angelovici, R., Yalovsky, S., and Ohad, N. (2004). Detection of protein-protein interactions in plants using bimolecular fluorescence complementation. Plant J 40, 419-427.

Caldelari, D., Sternberg, H., Rodriguez-Concepcion, M., Gruissem, W., and Yalovsky, S. (2001). Efficient prenylation by a plant geranylgeranyltransferase-I requires a functional CaaL box motif and a proximal polybasic domain. Plant Physiol 126, 1416-1429.

Chautard, E., Thierry-Mieg, N., and Ricard-Blum, S. (2009). Interaction networks: from protein functions to drug discovery. A review. Pathol Biol (Paris) 57, 324-333.

Chen, W.S., Chang, Y.C., Chen, Y.J., Teng, C.Y., Wang, C.H., and Wu, T.Y. (2009). Development of a prokaryotic-like polycistronic baculovirus expression vector by the linkage of two internal ribosome entry sites. J Virol Methods 159, 152-159.

Chen, X., Pham, E., and Truong, K. (2010). TEV protease-facilitated stoichiometric delivery of multiple genes using a single expression 
vector. Protein Sci 19, 2379-2388.

Clements, A., Johnston, K., Mazzarelli, J.M., Ricciardi, R.P., and Marmorstein, R. (2000). Oligomerization properties of the viral oncoproteins adenovirus E1A and human papillomavirus E7 and their complexes with the retinoblastoma protein. Biochemistry 39 , 16033-16045.

Dzivenu, O.K., Park, H.H., and Wu, H. (2004). General co-expression vectors for the overexpression of heterodimeric protein complexes in Escherichia coli. Protein Expr Purif 38, 1-8.

Fabian, J.R., Kimball, S.R., and Jefferson, L.S. (1998). Reconstitution and purification of eukaryotic initiation factor 2B (elF2B) expressed in Sf21 insect cells. Protein Expr Purif 13, 16-22.

Garcia, J.A., Riechmann, J.L., and Lain, S. (1989). Artificial cleavage site recognized by plum pox potyvirus protease in Escherichia coli. J Virol 63, 2457-2460.

Hanzlowsky, A., Jelencic, B., Jawdekar, G., Hinkley, C.S., Geiger, J.H., and Henry, R.W. (2006). Co-expression of multiple subunits enables recombinant SNAPC assembly and function for transcription by human RNA polymerases II and III. Protein Expr Purif 48, 215-223.

Himmler, G., Frank, S., Steinkellner, H., Ruker, F., Mattanovich, D., and Katinger, H.W. (1990). Detection of the trans activity of the plum pox virus Nla-like protease in infected plants. J Gen Virol 71 (Pt 7), 1623-1625.

Huppa, J.B., and Ploegh, H.L. (1997). In vitro translation and assembly of a complete T cell receptor-CD3 complex. J Exp Med 186, 393-403.

Kerrigan, J.J., Xie, Q., Ames, R.S., and Lu, Q. (2011). Production of protein complexes via co-expression. Protein Expr Purif 75, 1-14.

Krestine, G., Tanja, L.C., Michael, K.J., Flemming, M.P., and Karen, S. (2003). Interactions between plant RING-H2 and plant-specific NAC (NAM/ATAF1/2/CUC2) proteins: RING-H2 molecular specificity and cellular localization. Biochem. J. 371, 97-108.

Kummel, D., Muller, J.J., Roske, Y., Misselwitz, R., Bussow, K., and Heinemann, U. (2005). The structure of the TRAPP subunit TPC6 suggests a model for a TRAPP subcomplex. EMBO Rep 6, 787-793.
Neumann, D., Suter, M., Tuerk, R., Riek, U., and Wallimann, T. (2007). Co-expression of LKB1, MO25alpha and STRADalpha in bacteria yield the functional and active heterotrimeric complex. Mol Biotechnol 36, 220-231.

Patial, S., Chaturvedi, V.K., Rai, A., Saini, M., Chandra, R., Saini, Y., and Gupta, P.K. (2007). Virus neutralizing antibody response in mice and dogs with a bicistronic DNA vaccine encoding rabies virus glycoprotein and canine parvovirus VP2. Vaccine 25, 4020-4028.

Phizicky, E.M., and Fields, S. (1995). Protein-protein interactions: methods for detection and analysis. Microbiol Rev 59, 94-123.

Scheich, C., Kummel, D., Soumailakakis, D., Heinemann, U., and Bussow, K. (2007). Vectors for co-expression of an unrestricted number of proteins. Nucleic Acids Res 35, e43.

Selleck, W., Fortin, I., Sermwittayawong, D., Cote, J., and Tan, S. (2005). The Saccharomyces cerevisiae Piccolo NuA4 histone acetyltransferase complex requires the Enhancer of Polycomb $\mathrm{A}$ domain and chromodomain to acetylate nucleosomes. Mol Cell Biol 25, 5535-5542.

Shih, Y.P., Wu, H.C., Hu, S.M., Wang, T.F., and Wang, A.H. (2005). Self-cleavage of fusion protein in vivo using TEV protease to yield native protein. Protein Sci 14, 936-941.

Tundup, S., Akhter, Y., Thiagarajan, D., and Hasnain, S.E. (2006). Clusters of PE and PPE genes of Mycobacterium tuberculosis are organized in operons: evidence that PE Rv2431c is co-transcribed with PPE Rv2430c and their gene products interact with each other. FEBS Lett 580, 1285-1293.

Urcuqui-Inchima, S., Haenni, A.L., and Bernardi, F. (2001). Potyvirus proteins: a wealth of functions. Virus Res 74, 157-175.

Villemure, J.F., Savard, N., and Belmaaza, A. (2001). Promoter suppression in cultured mammalian cells can be blocked by the chicken beta-globin chromatin insulator 5'HS4 and matrix/scaffold attachment regions. J Mol Biol 312, 963-974.

Zheng, N., Perez Jde, J., Zhang, Z., Dominguez, E., Garcia, J.A., and Xie, Q. (2008). Specific and efficient cleavage of fusion proteins by recombinant plum pox virus Nla protease. Protein Expr Purif 57 , 153-162. 